\title{
Fresnel Diffraction by Deterministic Fractal Gratings: An Experimental Study ${ }^{1}$
}

\author{
D. Rodriguez Merlo, J. A. Rodrigo Martín-Romo, T. Alieva, and M. L. Calvo \\ Departamento de Óptica, Facultad de Ciencias Físicas, Universidad Complutense de Madrid, Madrid, 28400 Spain \\ e-mail: talieva@fis.ucm.es; mlcalvo@fis.ucm.es \\ Received December 20, 2002
}

\begin{abstract}
Experimental results are presented for light diffraction under the Fresnel regime by two types of fractal gratings: triadic Cantor bars and Vicsek fractals. It is shown that the evolution of the diffraction patterns in the Fresnel regions reveals the hierarchical structure of deterministic fractal gratings. (C) 2003 MAIK "Nauka/Interperiodica".
\end{abstract}

The main properties of Fresnel diffraction by regular fractal gratings have been studied theoretically in various publications [1-5], although not all of them have been experimentally verified.

In particular, it was shown analytically and by numerical simulations for various fractal gratings [3-5] that the Fresnel integral

$$
\begin{gathered}
U(\xi, z)=R^{z}[f(x)](\xi) \\
=\frac{1}{\sqrt{i \lambda z}} \int f(x) \exp \left(-i \frac{\pi}{\lambda z}(\xi-x)^{2}\right) d x
\end{gathered}
$$

of a homogeneous function $f(x)$

$$
f(x / m)=m^{\alpha} f(x),
$$

where $m$ is a scaling factor and $\alpha$ is a scaling exponent, is self-affine with respect to longitudinal $z$ and transversal $\xi$ coordinates

$$
U\left(\xi / m, z / m^{2}\right)=m^{\alpha} U(\xi, z) .
$$

The fractal structure is usually described by some iterative procedure, and relation (2) holds not for all $m$, but for some sequence $m^{k}$, where $k=1, \ldots, n$ and $n$ is a level of the fractal. Because of the finite $n$ and the finite size of real-world fractal structures, relation (2) transforms to

$$
f_{n}\left(m^{-k} x\right)=m^{k \alpha} f_{n-k}(x)
$$

and, therefore,

$$
U_{n}\left(\xi / m^{k}, z / m^{2 k}\right)=m^{k \alpha} U_{n-k}(\xi, z) .
$$

Another property of Fresnel diffraction by regular fractals is the invariance of the hierarchical structure of diffracted fractal fields [6]. Thus, if an input fractal grating $f_{n}(x)$ of level $n$ is constructed from the initiator

\footnotetext{
${ }^{1}$ This article was submitted by the authors in English.
}

$f_{0}(x)$ through a certain iterative procedure including affine transformations of $f_{0}(x)$ (scaling and shifting), then the complex amplitude of the diffracted field $U_{n}(\xi$, $z)=R^{z}\left[f_{n}(x)\right](\xi)$ can be generated by applying the same iterative procedure taken as the initiator $U_{0}(\xi, z)=$ $R^{z}\left[f_{0}(x)\right](\xi)$. Therefore, $U_{n}(\xi, z)$, as well as $f_{n}(x)$, can be built from $U_{n-k}(\xi, z)$ and $f_{n-k}(x)$, respectively, after $k$ iterations.

The goal of this paper is to observe and assert these properties experimentally. We study the evolution of the Fresnel diffraction patterns of fractal fields. The latter are generated after the free propagation of a plane wave through deterministic fractal amplitude gratings. Concretely, we consider two binary gratings: a triadic Cantor set and a Vicsek curve. These fractals were generated on a personal computer and then printed by an HP Laser 4P printer on transparencies. The size of the fractal amplitude gratings is $3.5 \times 2.5 \mathrm{~cm}$.

The optical system assembled for the experiments is displayed in Fig. 1. It consists of a $\mathrm{He}-\mathrm{Ne}$ laser (wavelength $\lambda=632.8 \mathrm{~nm}$, power $P=20 \mathrm{~mW}$ ), a pinhole 1 , a polarizer 2 used for control of the illumination level, a collimator lens $L 1$ (focal length $f_{1}=50 \mathrm{~cm}$ ), and a Hitachi KP 140 CCD camera connected to the computer. The spatial high-frequency resolution of the system is limited to 37 lines/mm (as obtained from the experimental MTF). Two additional lenses $L 2$ and $L 3$ with focal lengths $f_{2}=50 \mathrm{~cm}$ and $f_{3}=18 \mathrm{~cm}$, respectively, are used in the experiment for Vicsek fractal study in order to decrease the size of the initial grating by a factor of 3 before observation of the Fresnel diffraction and accommodate proper detection.

First, we study the Fresnel diffraction pattern produced by Cantor bars of level 5, partially shown in Fig. 2a (the horizontal width of the white bar is $0.2 \mathrm{~mm}$ ). The pattern is formed by the iterative procedure of dividing a bar into three equal segments and removing its middle third. Then, the scaling factor $m$ in 


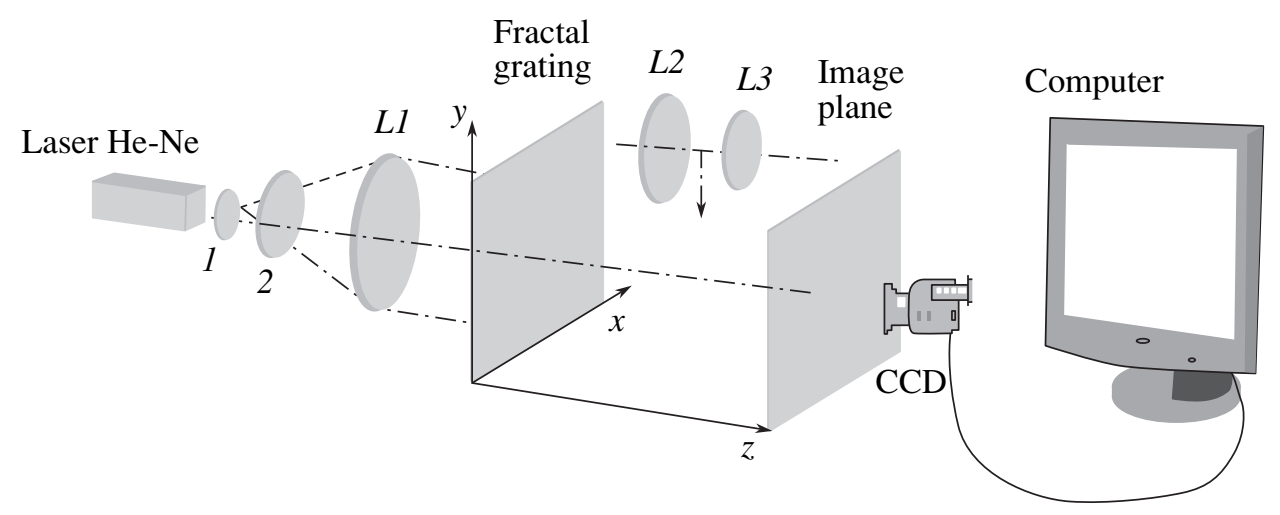

Fig. 1. Experimental setup used in the experiments. Additional lenses $L 2$ and $L 3$ are applied for observation of diffraction by the Vicsek fractal.

(a)

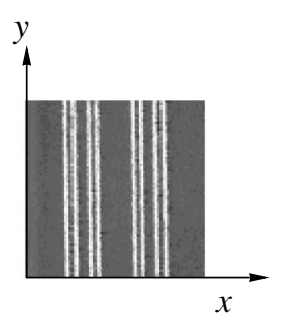

(b)

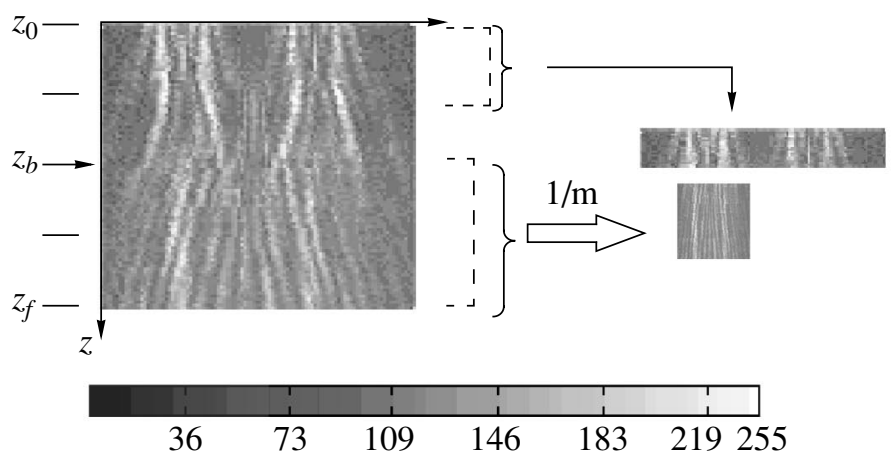

Fig. 2. (a) Part of the triadic Cantor set of level 5, registered by the CCD camera. (b) The hierarchical tree of the Cantor set, obtained from the observation of the intensity evolution of the diffractive patterns along the optical axis $z$. (c) Experimental verification of Eq. (5).

Eqs. (4) and (5) equals 3 . In reality, due to the quality of the printer used for preparation of the grating, $m=$ 2.8. This means that the initial bar is divided into 14 equal segments and 4 of them are removed from the middle.

Moving the CCD camera along the optical axis from the closest position to the object plane $(15 \mathrm{~cm})$ to the farthest allowed by the system $(177 \mathrm{~cm})$, we obtain a sequence of 40 Fresnel diffraction patterns. Selecting the same small region $\Delta y$ from every pattern and placing them consecutively in growing order of $z$, we build up an image (Fig. 2b) representing a part of a fractal tree, which reveals the hierarchical structure of the Cantor set. The gray scale indicates the intensity levels. One can observe that, during propagation in free space, the field associated with the fractal of level $n$ transforms into the field associated with the structure of the lower level $n-1$. The bifurcation point where this transformation occurs is indicated by $z_{b}=113 \mathrm{~cm}$ in Fig. 2b. From analysis of Fig. 2b, we can conclude that another bifurcation occurs at the distance $z_{0}=15 \mathrm{~cm}$ from the object plane. This is the first registered diffraction pattern due to the specifics of our experimental setup. The experimental observation of the evolution of the Fresnel diffraction patterns verifies Eqs. (4) and (5). Thus, the ratio between the distances where the similar intensity distributions are observed is $m^{2}=z_{b} / z_{0}=7.5$, which corresponds to the scaling factor $m=2.8 \approx 3$. Moreover, after rescaling the coordinate $x$ of the fractal tree for distances $z \in\left[z_{b}, z_{f}\right]$ by the factor $1 / m$, we obtain an image (Fig. 2c) that coincides with every two parts of the Cantor tree for distances $z \in\left[z_{0}, z_{1}\right]$.

Therefore, from analysis of the intensity distribution of the Fresnel patterns along the optical axis, information about the hierarchical structure of the regular fractal gratings and its scaling parameters can be derived.

In the second experiment, we demonstrate the invariance of the hierarchical structure of the diffractive patterns. Since this law holds for a complex field amplitude of the diffractive fields, its verification from the analysis of the intensity distributions measured in the 


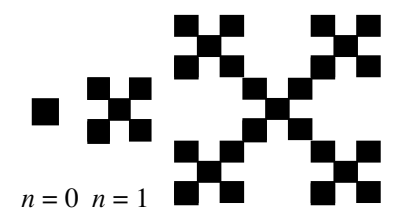

(a)

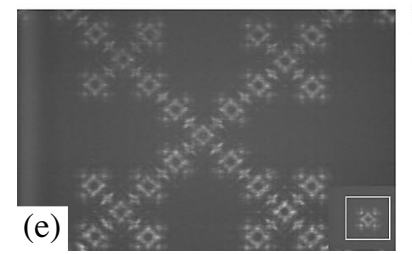

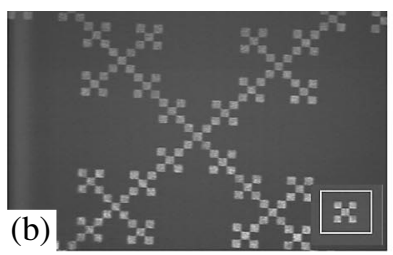

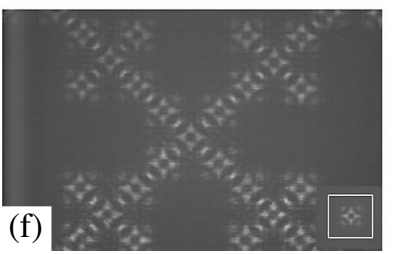

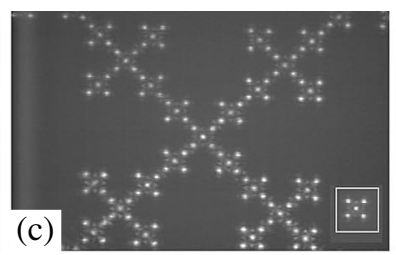

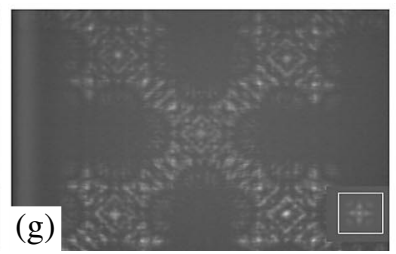

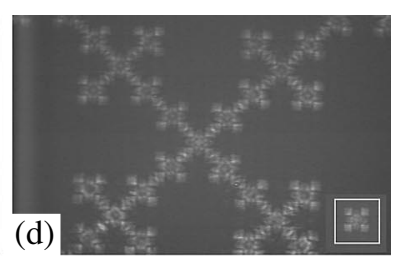

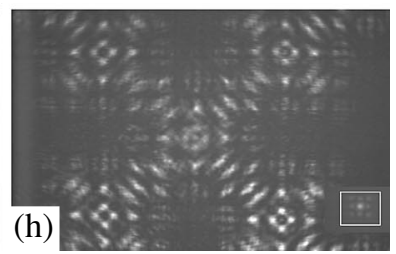

Fig. 3. (a) Successive stages of Vicsek fractal generation. (b-h) Fresnel patterns of the Vicsek fractal of level 4 and its generator (boxed) for the distances $0,2.5,4.6,7.3,10.3,18.3$, and 32.6 from the scaled object plane, respectively.

experiment is possible only for relatively small distances $z$, inside the near field.

The objects of our study are the Vicsek fractal of level $n=4$ and its generator $(n=1)$. The Vicsek fractal is generated starting from a square as an initiator and setting at every corner another square with the same dimensions, as described in Fig. 3a. The size of a side of initiator is $0.53 \mathrm{~mm}$. To decrease the size of the object by a factor of 3 , we used the additional lenses $L 2$ and $L 3$ shown in Fig. 1.

Figs. 3b-3h show a sequence formed by seven diffraction patterns for the following distances $(\mathrm{cm})$ from the scaled object plane: $0,2.5,4.6,7.3,10.3,18.3$, and 32.6. The evolution of the diffraction patterns for the generator is shown in the right corners inside the white boxes. It is observed that the diffracted image $\left|U_{4}(\xi, z)\right|^{2}$ can be reproduced from $\left|U_{1}(\xi, z)\right|^{2}$ after three iterations for distances $z \in[0,18.3] \mathrm{cm}$ (Figs. 3b-3g) by applying the same iterative procedure used for the generation of

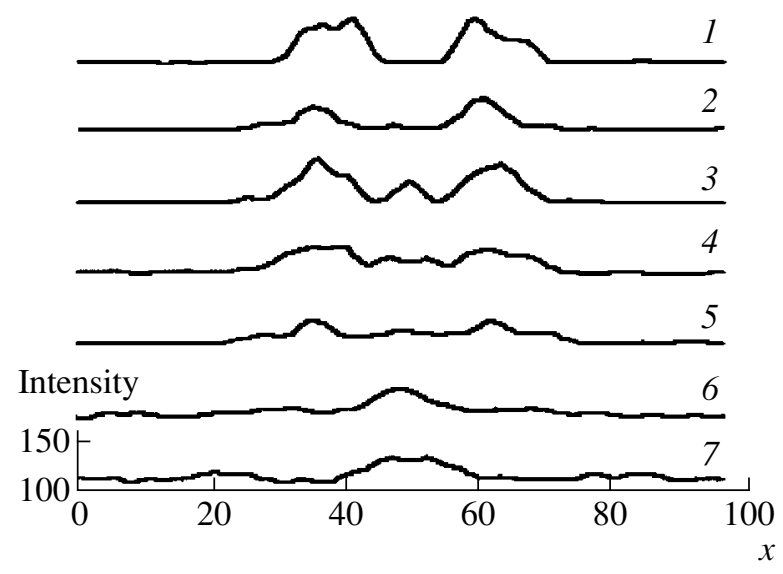

Fig. 4. Line profiles of the intensity distributions of the field diffracted by the generator along the $x$ axis for the distances $z=$ (1) 0 , (2) 2.5 , (3) 4.6, (4) 7.3, (5) 10.3, (6) 18.3, and (7) $32.6 \mathrm{~cm}$. the Vicsek fractal $f_{4}(x)$. For larger distances (Fig. 3h), due to the strong influence of the phase, the intensity patterns cannot display the general law for complex field amplitude. In Fig. 4, the profiles of the intensity distributions of the field diffracted by the generator $f_{1}(x)$ along the $x$ axis for the distances $z=0,2.5,4.6,7.3$, $10.3,18.3$, and $32.6 \mathrm{~cm}$, (curves $1-7$, respectively) are shown. One can see that the intensity of the first six line profiles (Fig. 4, curves $1-0$ ) is concentrated in the limited region of $x \in[30,70]$ normalized units, while, for $z=32.6 \mathrm{~cm}$, this intensity spreads over $x \in[10,90]$. Therefore, due to the interference phenomenon, the structure $\left|U_{1}(\xi, z)\right|^{2}$ cannot be observed in the pattern $\left|U_{4}(\xi, z)\right|^{2}$ (Fig. 3h). This fact indicates that it is not always possible to reveal the fractal properties of optical fields on the basis of only measurements of the intensity distribution.

\section{ACKNOWLEDGMENTS}

Grant no.TIC 2000-2791E from the Spanish Ministry of Science and Technology is acknowledged. T.A. would like to thank the Spanish Ministry of Education, Culture, and Sport for financial support (grant no. SB2000-0166).

\section{REFERENCES}

1. M. V. Berry, J. Phys. A 12, 781 (1979).

2. D. L. Jaggard and Y. Kim, J. Opt. Soc. Am. A 4, 1055 (1987).

3. Y. Sakurada, J. Uozumi, and T. Asakura, Pure Appl. Opt. 1, 29 (1992).

4. Y. Sakurada, J. Uozumi, and T. Asakura, Pure Appl. Opt. 3, 371 (1994).

5. T. Alieva and F. Agullo-Lopez, Opt. Commun. 125, 267 (1996).

6. T. Alieva and M. L. Calvo, in Proceedings of the 19th Conference of International Commission for Optics on Optics for the Quality of Life (Firenze, Italy, 2002), p. 337. 